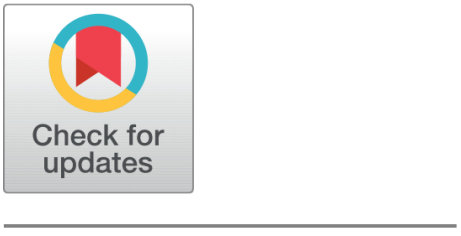

G OPEN ACCESS

Received: 10.12 .2021

Accepted: 30.12 .2021

Published: 21.01.2022

Citation: Newton PC, Felix AT (2022) ETX-Aware Energy-Efficient Algorithm to Reduce Packet Retransmissions in the Internet of Things. Indian Journal of Science and Technology 15(1): 28-43. https:// doi.org/10.17485/IJST/v15i1.2100

* Corresponding author.

athomasfelix@gmail.com

Funding: None

Competing Interests: None

Copyright: @ 2022 Newton \& Felix. This is an open access article distributed under the terms of the Creative Commons Attribution License, which permits unrestricted use, distribution, and reproduction in any medium, provided the original author and source are credited.

Published By Indian Society for Education and Environment (iSee)

ISSN

Print: 0974-6846

Electronic: 0974-5645

\section{ETX-Aware Energy-Efficient Algorithm to Reduce Packet Retransmissions in the Internet of Things}

\author{
P Calduwel Newton ${ }^{1}$, A Thomas Felix ${ }^{2 *}$ \\ 1 Department of Computer Science, Government Arts College, Trichy-22, India, Affiliated to \\ Bharathidasan University, Tiruchirappalli \\ 2 Department of Computer Science, Government Arts College, Kulithalai, India, Affiliated to \\ Bharathidasan University, Tiruchirappalli
}

\section{Abstract}

Objectives: An energy-efficient optimum path selection to reduce the number of packet retransmissions in a path is proposed in this paper using a new metric New-Expected Transmission Count (N-ETX), Residual Energy (RE), and Path Objective Function (POF). Methods: Energy efficiency, packet retransmission, and RE is considered while making the best choice of the optimum path. The refined N-ETX metric is employed to measure the Packet Delivery Ratio (PDR) of a node. If the PDR ratio is higher, this node is included in the optimum path set. The proposed algorithm avoids the inclusion of critical nodes such as dead nodes if the RE of the nodes is lower than the predefined Energy Threshold $\left(\mathrm{E}_{T H R}\right)$. The Unicast message reply to strategy significantly decreases the quantity of control message overhead. The T-test's Degree of Variance and Degree of Independence is used to discover the energy-efficient optimum path. It is demonstrated through performance outcomes that the obtained Quality of Service (QoS) parameters for the proposed algorithm stands superior to the state-of-art protocols. Findings: An algorithm established on N-ETX and RE metrics is proposed, and the discussions have shown that this approach is energy efficient. This metric decreases the quantity of packet retransmission effectively for a path. As an outcome, the sensor's higher energy consumption is reduced. The proposed algorithm outperforms the current algorithm by decreasing energy consumption, reducing the quantity of packet retransmission, improving the PDR ratio, and enhancing the network life expectancy. The simulation outcome established that energy consumed is decreased by $19.07 \%$, RE of the nodes is increased by $10.51 \%$, PDR ratio is increased to $98 \%$, and network lifespan is increased by $30.54 \%$. Novelty: Internet of Things (IOT) gadgets are bound by hardware limits regarding computation, memory, and energy proficiency. To effectively transmit data packets, every loT device must have a routing communications protocol that is easy to create. IoT devices necessitate the demand for self-adaptive routing algorithms. During network operation, packet energy dissipation during the broadcast and reception process is significantly higher when assessed to other 
energy-consuming processes like sensing, data processing, etc.

Keywords: LOADng; Energy Efficiency; ETX; Packet Retransmission; Residual Energy

\section{Introduction}

Sensors, electronics, software, and a network make up the IoT to gather or share data and perform specific tasks. The corporate and scientific communities have noticed its seemingly limitless potential, business, and research opportunities. The IoT has many benefits, but it also has certain drawbacks. Hardware limits such as computation, memory, and power consumption bind IoT devices. The entire internet traffic is overloaded primarily due to the recipient's information shield, raising the likelihood of packet loss. Furthermore, energy use and production have environmental consequences, particularly the threat posed by global climate change caused by greenhouse gas emissions from the global energy system. The key problem is how the existing sensor network's operating standards are defined for the prolonged duration of the battery by minimizing energy dissipation and improving the network lifespan. Another problem is the high packet loss during the retransmission of packets among two nodes. Also, the network operational cost is higher ${ }^{(1)}$.

Energy proficiency is a highly critical component because it determines the network's lifespan. The data transfer methods are crucial, consuming $70 \%$ of total energy. Moreover, finding the most efficient energy paths between sensor nodes and base stations is critical. The optimization concepts will help locate the optimum paths and reduce network exhaustion ${ }^{(2)}$. The key goals are reducing energy utilization and energy proficiency at both the device and network levels. Growing importance in IoT research and development has focused on these two areas in recent years: (a) designing and implementing energy efficiency techniques and mechanisms at the device level and (b) developing theoretical and empirical models for IoT energy utilization and battery life $^{(3)}$. But these aspects of energy proficiency and lowering energy utilization are addressed only to some extent by recent studies.

The success story of IoT applications hangs on reliable data transfer between sensors and servers. Each IoT application has unique QoS requirements. To communicate effectively, enormous IoT will need 99.99999 percent ultra-reliability. Notably, vital applications involving human safety, such as driving and healthcare, require this level of assurance. The applications have to provide data quickly ${ }^{(4)}$. Reactive routing offers minimal routing overhead since it doesn't require route management when no information is transmitted that minimizes routing data, reducing traffic and costs. Frequent node mobility is a primary concern that can break the route and end the communication. Under this situation, node migration uses more energy and disrupts the network. Overload traffic consumes considerable energy and degrades network performance regarding QoS. Reactive protocols use less power, but they initiate a discovery process to find another route when a route is lost. Link failure adds to network burden and wastes energy through route discovery. These degrade network performance by raising the load, decreasing throughput, and, therefore, the PDR ratio. So, a method that saves energy and improves performance is sought. This method supports a single optimum path for data transmission and doesn't support the dynamic environment of node mobility ${ }^{(5)}$.

Energy proficiency is vital for long-term network uptime, especially for devices installed in severe ecosystems where battery replacement and charging are impractical. Thus, energy-efficient routing methods manage device energy usage and increase network lifespan. The demerit of this method is that it consumes energy, causing higher energy consumption during the data aggregation process every round ${ }^{(6)}$. Heterogeneous sensor network-enabled applications have diverse performance requirements like 
low energy usage and low latency. Clustering helps improve dependability and energy proficiency. However, this method causes overhead among cluster heads and the devices nearer to the IoT gateway server. Also, existing route selection approaches route real-time and non-real-time packets utilizing the same path, thus reducing the system performance. Also, this method lacks low operational cost, security, reliability, and efficient energy management ${ }^{(7)}$.

Routing selects the most efficient path from the sensor node to the base station. The network operates longer using lowenergy packet transfer and routing strategies. The reactive routing communications protocol is an excellent energy-efficient data transmission method because it seeks the most efficient way ${ }^{(8)}$. This method lacks a technique to retransmit the data packets in case of packet loss and increases the data communication delay. For achieving efficient routing, sensor nodes must self-organize. During network operation, packet energy consumption during the broadcast and reception process is significantly higher than other energy-consuming processes like sensing, data processing, etc. Each retransmission is treated as a fresh transmission, and as the required number of packet retransmissions increases, the network's lifespan can suffer significantly. The volume of transmitted packets can also increase the packet collision probability. Hence, each IoT device must have a routing communications protocol that is simple to create, effective in packet transmissions, and energy-efficient.

Lightweight On-Demand Ad-hoc Distance Vector routing protocol-Next Generation (LOADng) is a reactive protocol for IoT networks published in 2011 by Clausen et al., ${ }^{(9)}$. LOADng proposes modifications to the Ad-hoc On-demand Distance Vector (AODV) protocol to make it reliable and lightweight for Low Power and Lossy Networks (LLN). Still, its fundamental flaws are route establishment latency, inefficient flooding, packet collision due to packet retransmission, and high overhead, as expressed in ${ }^{(10)}$. The IPv6 Routing Protocol for Low-Power and Lossy Networks (RPL) does not correctly enable mobility. It also limits point-to-point traffic, multicast data forwarding, and memory use. LOADng-IoT-Mob is an alternate to the RPL protocol ${ }^{(11)}$. This scheme results in increased memory usage in the mobile environment and increased network overhead.

Sobral et al., ${ }^{(12)}$ designed LOADng-IoT after examining IoT scenarios with various traffic and node patterns. It introduced a route cache and route discovery technique. It decreases the total broadcasts needed to locate an internet path, lowering overhead and energy utilization. The authors failed to emphasize the performance decrease caused by network topology changes as nodes relocate. Imtiaz A. Halepoto et al., ${ }^{(13)}$ conducted an experimental investigation with multiple interfaces aimed at IoT devices. Connection loss is typical in such gadgets, necessitating fast recovery of the connection and data. They discussed two retransmission policies large bandwidth and small bandwidth. The retransmission policies increased the packet loss ratio in the primary path and caused less throughput. This scheme also failed to recover from data loss and connection failure. Low Power Wide Area Network (LoRaWAN), according to Biswajit Paul ${ }^{(14)}$, uses seven uplink spreading parameters. LoRaWAN supports four cyclic coding speeds for forwarding error correction. LoRaWAN also enables many bandwidths and data-rate options. Node activity rate and collision probability rate decide the packet loss risk in systems. However, this technique failed to address the escalating packet retransmission due to the high packet error rate. It also resulted in high energy consumption due to packet loss and high packet collision probability.

Zhang X et al., ${ }^{(15)}$ developed a cell-free IoT to facilitate massive data communication between system nodes. They built a cell-free IoT energy efficiency optimization model. This method analyses the functioning of the cell-free IoT and handles the system's energy consumption. But this method failed to deliver the node's energy stagnation crisis. Ansere et al., ${ }^{(16)}$ suggested resource allocation. The electricity distribution problem and user selection are split into two sub-problems using Lagrangian dual decomposition and the KM algorithm. They presented an effective joint resource allocation technique to enhance energy efficiency. But this scheme failed to address the energy consumption related to channel uncertainty. According to Yasmin Fathy et al., ${ }^{(17)}$, high communication costs are caused by the constant transmission of large volumes of data between sensor and sink nodes. Environmental monitoring applications require more energy. Reducing data transit amid nodes can conserve energy and boost network lifespan, especially for battery-powered nodes. To mitigate overall data communication and transmission connecting sensor nodes, they introduced the Adaptive Method for Data Reduction (AMDR) to recreate accuracy boundary with original data. This method failed to detect event patterns at base stations based on multi-dimensional sensor data, which results in unanticipated high energy consumption.

Alireza Izaddoost et al., ${ }^{(18)}$ explained how remote IoT nodes must deliver data to the server across numerous hops. Picking the forwarding node with peak energy magnitude from a list may decrease energy utilization and intensify the node's operational time. But this method failed to focus on the data balance during inter-cluster routing through different paths. This resulted in increased energy consumption. The wireless connection with a server and other nodes can waste approximately $70 \%$ of the sensor's energy; hence, solutions must be discovered ${ }^{(19)}$. In this scheme, the sink node is placed on the human body to be close to one another. But when the sink is moved away from the body and placed somewhere else, a method has to be devised to minimize the energy utilization by selecting the optimum path and reducing packet retransmission. The routing procedures are vital for assuring optimal sensor communication and increasing network lifespan by lowering energy usage. ${ }^{(20)}$ describes a method for allocating and routing IoT resources in multi-cloud situations. The authors suggested an Energy-efficient and 
Congestion-aware Resource allocation and Routing protocol (ECRR) for IoT networks. But the drawback of this method is that it generates HELLO messages at regular intervals for path discovery, resulting in overhead and high energy consumption.

$5 \mathrm{G}$ networks bring fresh designs and customizable support for new apps. However, IoT applications demand small latency, high energy economy, and long-range communication capability ${ }^{(21)}$. Julio C.S. dos Anjos et al., ${ }^{(22)}$ proposed a dynamic cost model with task scheduling, cloud computing, and edge computing. This method accounts for task handling and data communication costs by correlating each layer's allocation strategy. The drawback of this method is that it can handle only lower data size. When a higher data size is used, it results in high energy dissipation proportionate to the size of the data. Jaiswal et al., ${ }^{(23)}$ developed an effective routing algorithm. The next-hop node's reliability, longevity, and traffic intensity decide the optimum path. This technique saves energy, improves PDR, and extends network lifetime. The demerit of this technique is underperformed during parallel transmissions of data resulting in packet loss and frequent retransmissions, and high energy consumption. Also, it doesn't support implementation in heterogeneous networks. ${ }^{(24)}$ presents a method to stabilize the parameters like path reliability and energy consumption. But this method failed in integrated data security, resulting in distorted energy consumption of nodes. ${ }^{(25)}$ introduces a method to improve the overall energy utilization of nodes. But this method has low throughput and an increase in packet drop ratio. This resulted in frequent retransmission of packets between nodes resulting in high energy consumption, and less RE. ${ }^{(26)}$ proposed a method to resolve the issues of dynamic IoT networks. Data mining is employed for user authentication. This method works well with a single sink node, but it results in higher energy consumption when multiple sink nodes are used. ${ }^{(27)}$ proposed a method to establish optimum paths centered on both energy of the nodes and trust. But this scheme has a high error rate of packets, resulting in frequent retransmission of packets, thus increasing energy utilization and reducing RE.

The significant merits and demerits of the recent works are charted in Table 1.

Table 1. Merits and Demerits of Previous works

\begin{tabular}{|c|c|c|c|c|}
\hline Citations & Authors & $\begin{array}{l}\text { Protocols/ } \\
\text { Schemes Used }\end{array}$ & Advantages & Disadvantages \\
\hline (1) & Arul R et al., & IDA-OEP & $\begin{array}{l}\text { - Low energy utilization } \bullet \text { Energy optimization } \\
\text { - Reduces time }\end{array}$ & $\begin{array}{l}- \text { Packet loss during retrans- } \\
\text { mission } \bullet \text { Data reliability } \bullet \\
\text { Operational cost }\end{array}$ \\
\hline (5) & Patel J et al., & GA-AOMDV & $\begin{array}{l}\text { - Extended lifespan of nodes } \bullet \text { Less energy } \\
\text { consumption }\end{array}$ & $\begin{array}{l}\text { - Single path route } \bullet \text { Node } \\
\text { mobility }\end{array}$ \\
\hline (6) & $\begin{array}{l}\text { Mutombo VK et } \\
\text { al., }\end{array}$ & EER-RL & $\begin{array}{l}\text { - Cluster-based reinforcement learning } \bullet \\
\text { Extends network lifetime } \bullet \text { Energy efficient }\end{array}$ & $\begin{array}{l}\text { - High energy utilization for } \\
\text { data aggregation }\end{array}$ \\
\hline (7) & Nayagi DS et al., & REERS & $\begin{array}{l}- \text { Heterogeneous network } \bullet \text { Cluster-based com- } \\
\text { munication } \bullet \text { Multi-path transmission }\end{array}$ & $\begin{array}{l}\text { - Store and process data } \\
\text { in the cloud environment } \bullet \\
\text { Security concerns }\end{array}$ \\
\hline (8) & Zhang S et al., & UCB-CoAP & $\begin{array}{l}\text { - Minimized transmission delay } \bullet \text { Minimized } \\
\text { packet loss ratio }\end{array}$ & $\begin{array}{l}- \text { Increases transmission } \\
\text { delay } \bullet \text { No retransmission } \\
\text { technique }\end{array}$ \\
\hline (10) & Zikria YB et al., & LOADng & - Reactive protocol & $\begin{array}{l}\bullet \text { Increased latency } \bullet \text { Flood- } \\
\text { ing } \bullet \text { Packet collision } \bullet \text { High } \\
\text { overhead }\end{array}$ \\
\hline (11) & Sobral JVV et al., & $\begin{array}{l}\text { LOADng-IoT- } \\
\text { Mob }\end{array}$ & $\begin{array}{l}\bullet \text { Supports topology changes } \bullet \text { Efficient network } \\
\text { performance } \bullet \text { Reduced energy consumption } \bullet \\
\text { High PDR }\end{array}$ & $\begin{array}{l}\text { - Increased control overhead } \\
\text { - Increased memory usage }\end{array}$ \\
\hline (12) & Sobral JVV et al., & LOADng-IoT & $\begin{array}{l}\bullet \text { Unicast transmission } \bullet \text { New error code } \bullet \text { Low } \\
\text { latency } \bullet \text { Reduced packet collisions }\end{array}$ & $\begin{array}{l}\text { - Performance degradation } \\
\text { in increased network density }\end{array}$ \\
\hline (13) & Halepoto IA et al., & SCTP & • Quick delivery • Retransmissions policy & $\begin{array}{l}\text { - Handling multiple streams } \\
\text { - Connection failure }\end{array}$ \\
\hline (14) & Paul B & LoRaWAN & - Increase in network lifespan $\bullet$ Increase in PDR & $\begin{array}{l}- \text { High packet collision } \bullet \text { The } \\
\text { high packet error rate }\end{array}$ \\
\hline (15) & Zhang X et al., & ABNFO & - Efficient resource allocation $\bullet$ Reliability & - Energy stagnation \\
\hline (16) & Ansere JA et al., & JPAUS & $\begin{array}{l}- \text { Energy efficient } \bullet \text { Less transmission power } \bullet \\
\text { Superior resource allocation }\end{array}$ & - Channel uncertainty \\
\hline (17) & Fathy Y et al., & AM-DR & $\begin{array}{l}- \text { Reduced energy utilization } \bullet \text { Reduced com- } \\
\text { munication cost } \bullet \text { Prolonged network lifetime }\end{array}$ & $\begin{array}{l}\text { - Multi-dimensional sensor } \\
\text { data }\end{array}$ \\
\hline
\end{tabular}

Continued on next page 


\begin{tabular}{|c|c|c|c|c|}
\hline \multicolumn{5}{|c|}{ Table 1 continued } \\
\hline (18) & Izaddoost A et al., & $\begin{array}{l}\text { Data transmis- } \\
\text { sion model }\end{array}$ & $\begin{array}{l}\text { - Balances workload } \bullet \text { Increases network stabil- } \\
\text { ity } \bullet \text { Less packet loss }\end{array}$ & $\begin{array}{l}\text { - Data-routing unbalance } \bullet \\
\text { Inter-cluster routing }\end{array}$ \\
\hline (19) & Khan RA et al., & $\mathrm{RK}$ & $\begin{array}{l}- \text { Single and multi-hop communication } \bullet \text { Less } \\
\text { energy consumption } \bullet \text { Stability } \bullet \text { Minimized } \\
\text { path loss }\end{array}$ & - Placement of sink node \\
\hline (20) & Praveen KV et al., & ECRR & $\begin{array}{l}- \text { Congestion conscious resource allocation } \bullet \\
\text { Scalability } \bullet \text { High network lifetime } \bullet \text { High } \\
\text { throughput }\end{array}$ & $\begin{array}{l}\text { - Self-organization } \bullet \text { HELLO } \\
\text { messages }\end{array}$ \\
\hline (22) & $\begin{array}{l}\text { Dos Anjos JCS et } \\
\text { al., }\end{array}$ & TEMS & $\begin{array}{l}- \text { Minimize idle CPU energy consumption } \bullet \\
\text { Less CPU processing time } \bullet \text { Local and remote } \\
\text { computing simultaneously } \bullet \text { Decrease in energy } \\
\text { utilization }\end{array}$ & $\begin{array}{l}\text { - Increase in number of task } \\
\text { allocations to a remote server } \\
\text { - Smaller data size }\end{array}$ \\
\hline (23) & Jaiswal K et al., & EOMR & $\begin{array}{l}\bullet \text { Minimized energy utilization per bit } \bullet \text { Energy } \\
\text { efficient } \bullet \text { Multi-path routing } \bullet \text { Less end-to-end } \\
\text { delay } \bullet \text { High PDR }\end{array}$ & $\begin{array}{l}\text { - Low PDR for parallel trans- } \\
\text { mission • Homogeneous net- } \\
\text { work }\end{array}$ \\
\hline (24) & $\begin{array}{l}\text { Nivedhitha } \mathrm{V} \text { et } \\
\text { al., }\end{array}$ & DMEERP & $\begin{array}{l}\text { - Less packet energy consumption • High } \\
\text { energy efficiency } \bullet \text { Increased network lifetime }\end{array}$ & - Less security \\
\hline (25) & $\begin{array}{l}\text { Maheshwari P et } \\
\text { al., }\end{array}$ & $\mathrm{BOA}$ and $\mathrm{ACO}$ & $\begin{array}{l}- \text { Reduced energy utilization } \bullet \text { Increased net- } \\
\text { work lifetime } \bullet \text { High performance }\end{array}$ & $\begin{array}{l}- \text { Lower throughput } \\
\text { Increase in Packet drop ratio }\end{array}$ \\
\hline (26) & Sujanthi S et al., & SecDL & - Improved QoS • Energy efficient $\bullet$ Security & - Multiple sink nodes \\
\hline (27) & Shende DK et al., & CrowWhale-ETR & $\begin{array}{l}- \text { Minimum delay } \bullet \text { Maximum throughput } \bullet \\
\text { Multicast routing }\end{array}$ & - High error rate \\
\hline $\begin{array}{l}\text { [Proposed } \\
\text { Study] }\end{array}$ & $\begin{array}{l}\text { Calduwel Netwon } \\
P \text { et al., }\end{array}$ & ETALGOR & $\begin{array}{l}- \text { Low energy consumption } \bullet \text { Less packet } \\
\text { retransmission } \bullet \text { High residual energy } \bullet \text { High } \\
\text { PDR } \bullet \text { Improved network lifespan }\end{array}$ & - Unbalanced load for a node \\
\hline
\end{tabular}

So, reducing IoT devices energy dissipation and reducing packet retransmission is good objective. The recent literature lacks the aspects of energy proficiency, optimized packet delivery, minimized packet retransmission, and mobility assistance repeated Route Request (RREQ) broadcast for path discovery which encouraged this work. The proposed study addresses the shortcomings in previous studies $(2,5,8,10,14,17,20,25)$, by reducing data packet retransmission between nodes, decreasing nodelevel energy consumption, decreasing device energy consumption, increasing PDR ratio, increasing RE, and increasing network lifespan.

\section{Proposed Work}

QoS is meant to prioritize energy usage, throughput, packet loss, end-to-end delay, latency, and jitter. Innovative applications and services benefit from QoS, as is the prevention of packet congestion. Reactive routing communications protocol creates routes only when an originator node requests one. Reactive routing communications protocols provide low CPU and memory overhead, low network utilization and avoid routing loops by employing destination sequence numbers. For Peer-to-Peer (P2P) communication between nodes with inadequate hardware and energy, the LOADng was developed. This protocol avoids the purpose of the AODV protocol's HELLO messages. But the major drawback is the repeated RREQ broadcast for path discovery procedure, increasing energy utilization. Traditionally, LOADng employed Hop Count (HC) as the default path discovery metric. However, the HC didn't consider the shortcomings of nodes, thus reducing the network's lifespan.

The Internet Engineering Task Force (IETF) defined hop-count as a typical routing parameter to detect the shortest link. This parameter's flaw ignores energy expenditure, channel bandwidth, and packet failure. This causes a high rate of packet failure and performance degradation. The ETX routing metric improves network speed and lowers packet retransmissions. ETX seeks paths with high throughput, few hops, and low PDR. The ETX parameter specifies the size of transmissions necessary to transfer a packet effectively. The equation for a link's ETX is given in (1),

$$
\mathrm{ETX}=\frac{1}{D_{f *} D_{r}}
$$

where $\mathrm{D}_{f}$ - the forward PDR ratio and $\mathrm{D}_{r}$ - the reverse PDR ratio. A path's cumulative ETX is the summation of the ETX for each of its links.

Each node broadcasts dedicated Link Probe Packets (LPP) of specified size periodically (usually 1 second) to its neighbors. Each LPP packet contains the reception ratio from each neighbor. Paths with higher throughput and smaller hops are favored 
based on delivery ratios. The optimum path's PDR ratio is computed. The ETX chooses a longer way with smaller hops if the optimum path has too many hops. It also calculates loop-free paths and measures link-layer losses. The network remembers the amount of link probe packets obtained from neighbor nodes and calculates the ETX measure. Receiving LPP packets from neighbors increases a node's number of LPP packets. The neighbor's number of LPP packets is updated. The cumulative sum of a path's links determines its ETX.

The proposed ETX Aware energy-efficient ALGORithm (ETALGOR) presents a metric denoted as N-ETX, the refined form of traditional ETX that is specified by equation (2),

$$
\mathrm{N}-\mathrm{ETX}=\frac{1}{\left(D_{f} * c\right)+\left((1-c) * D_{r}\right)}
$$

where " $\mathrm{c}=\sigma^{2}$ ", $\mathrm{c}$ is a factor, and $\sigma^{2}$ is the Variance of the packets successfully delivered.

Adding the N-ETX metric to the proposed ETALGOR requires several steps. The N-ETX field is included in each routing entry. If there are multiple paths from the origin to the target, the optimum path has the smallest N-ETX value. If more than one path has the identical N-ETX value, the path with the fewest hops is chosen. The N-ETX value is tagged onto the RREQ control message and Route Reply (RREP) control message. Each node updates the N-ETX field when RREQ is sent. A node's $\mathrm{N}$-ETX is the cumulative sum of N-ETX from previous nodes.

The source sends an RREQ message to the target node with packets to deliver when no route entry for the target node exists in the routing database. Consider node "S" sending packets to node "D". "S" broadcasts RREQ with N-ETX metric set to 0. It then calculates the N-ETX value to the neighboring node that sent the RREQ with the formerly obtained Forward LPP Count and Reverse LPP Count. The node updates its routing table, N-ETX value, and the RREQ received. The routing table is updated when the target node "D" receives RREQ. Here, the routing table's N-ETX is equal to the cumulative N-ETX of a path. Node “D” delivers a Unicast RREP to the origin, resetting N-ETX to 0 . The Unicast RREP follows the identical path till it reaches the origin, broadcasting data packets. Hence, there are fewer intermediate RREP's and thus more occasional control messages. Upon receiving the initial RREP message, the source begins transmitting data packets. The originator routing table is updated with the better path when receiving an additional RREP control message that contains a better N-ETX value.

The control messages of ETALGOR are identical in structure. The router checks if the message is acceptable before processing it on receiving a message. Figure 1 illustrates the ETALGORs' RREQ message format.

\begin{tabular}{|c|c|}
\hline \multicolumn{2}{|l|}{ RREQ Message Format } \\
\hline Field & Description \\
\hline addr-length & Defines the length of the addresses used by originator and destination nodes \\
\hline seq-num & $\begin{array}{c}\text { Indicates the sequence number that uniquely identifies each message } \\
\text { generated by the originator node }\end{array}$ \\
\hline metric-type & Determines the type of metric used by the message originator node \\
\hline route-metric & Defines the value of the route metric of the path \\
\hline hop-count & Indicates the number of hops that the message has traversed \\
\hline hop-limit & Indicates the maximum number of times that a message can be forwarded \\
\hline originator & Specifies the address of the message originator \\
\hline destination & Specifies the address of the message destination \\
\hline n-etx & Refined Expected Transmission Count of a node \\
\hline battery-level & Current battery level of a node \\
\hline
\end{tabular}

Fig 1. ETALGOR Control Messages with N-ETX metric and Battery level parameter

The proposed ETALGOR is outlined as follows,

ETALGOR: A Proposed Algorithm

Input: A Network including "N" nodes, Source "S" and Destination "D"

Output: An optimum path from "S" node to " $D$ " node

Initialize: $\mathrm{N}-\mathrm{ETX} \leftarrow 0$

$\mathrm{HC} \leftarrow 0$

$\mathrm{E}_{T H R \leftarrow 5} \%$ of initial-energy of a node

$\mathrm{RE}_{N} \leftarrow$ Current $\mathrm{RE}$ of a node

$\mathrm{RE}_{P} \leftarrow$ Cumulative sum of current RE of a path

Parameters:

POF () - Path Objective Function

N-ETX - Refined ETX

HC - Hop Count 


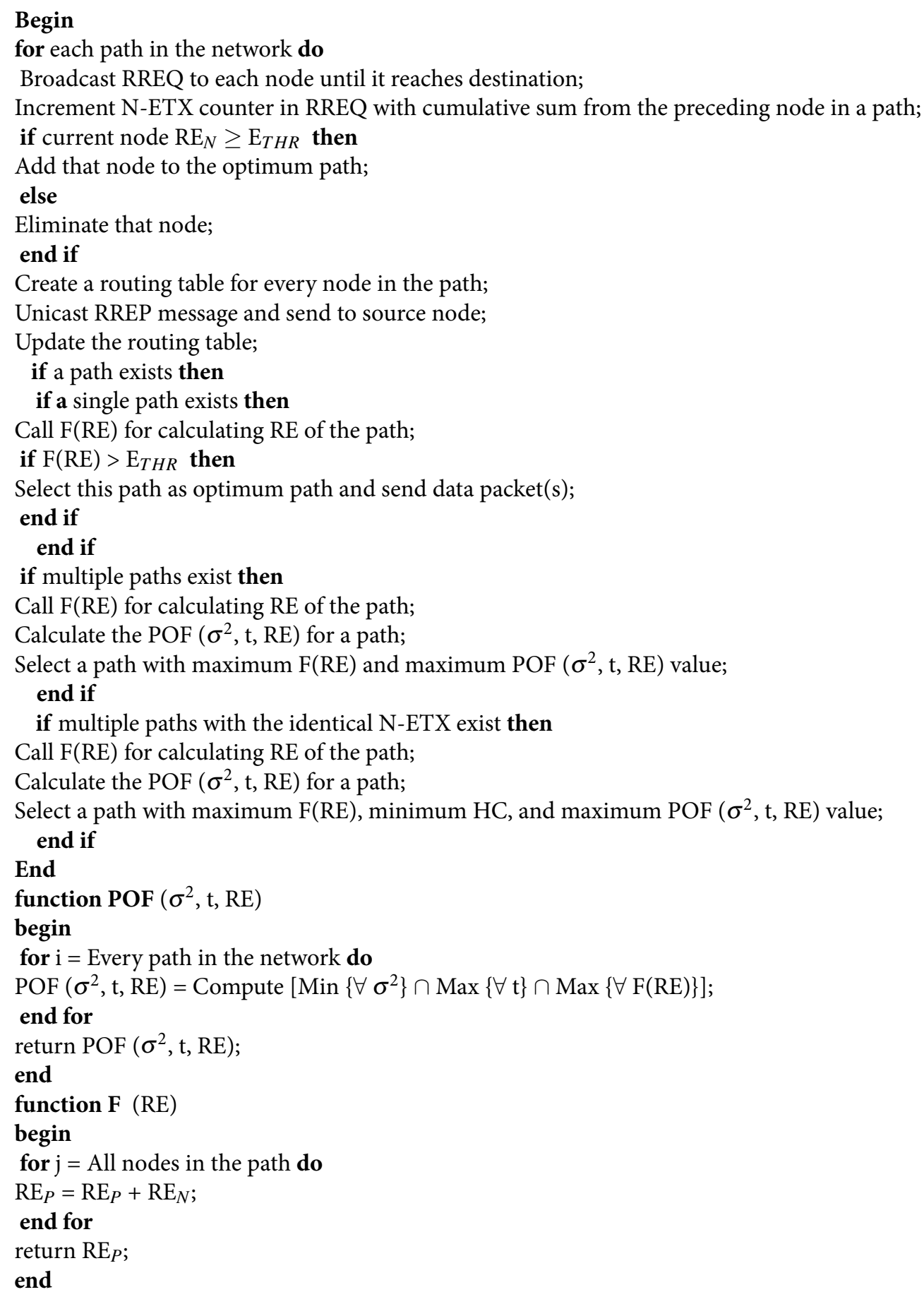

The ETALGOR selects an originator and target. The originator then broadcasts RREQ to the target. The originator sets N$\mathrm{ETX}=0$. When RREQ arrives at the neighboring node, increment the N-ETX counter. The N-ETX value is the sum up of the previous and current nodes. If the $\mathrm{RE}$ is larger than the $\mathrm{E}_{T H R}$, which equals $5 \%$ of the initial energy of a node, it is included in the optimum path. Otherwise, retransmission occurs, wasting energy, and are refused. The ETALGOR sends an RREP to the origin when RREQ arrives. Now update the paths' routing table. If just one option exists, compute its RE and if exceeds the $\mathrm{E}_{T H R}$, choose this path as the optimum path. Calculate N-ETX and the POF $\left(\sigma^{2}, \mathrm{t}, \mathrm{RE}\right)$ if numerous paths exist. The ETALGOR finds a path with the smallest N-ETX, maximum RE, and maximum POF $\left(\sigma^{2}, t, R E\right)$. Compare the path's residual energy if many paths have the identical N-ETX value. The ETALGOR finds the optimum path by minimizing HC and maximizing POF $\left(\sigma^{2}, t, R E\right)$. The path with the smallest N-ETX, highest RE, and highest POF $\left(\sigma^{2}, t, R E\right)$ value is consistently desired as the 
most energy-efficient path. The ETALGOR avoids paths that contain crucial nodes with $\mathrm{RE} \leq \mathrm{E}_{T H R}$. This approach avoids dead nodes, reduces unnecessary packet retransmission, and saves energy.

\subsection{Energy Model of the ETALGOR}

The nodes perform the operations of sensing, broadcast, and reception of data. Energy is consumed for all three operations. When a packet is transmitted from the originator to the target, they consume energy. The sender consumes more energy, unlike the receiver, to send packets and augment the signal over the distance.

The energy consumption of a node is computed as follows,

$$
E_{C}=E_{T X}+E_{R X}+E_{I D L E}+E_{C P U}+E_{C A}
$$

where Energy Consumed $\left(\mathrm{E}_{C}\right)$ is defined by transmission energy $\left(\mathrm{E}_{T X}\right)$, reception energy $\left(\mathrm{E}_{R X}\right)$, idle state energy $\left(\mathrm{E}_{I D L E}\right), \mathrm{CPU}$ active mode energy dissipation $\left(\mathrm{E}_{C P U}\right)$, and channel assessment energy $\left(\mathrm{E}_{C A}\right)$ and updates the RE. In the first order radio energy model, the transmission and reception energy $\mathrm{E}_{T X}$ and $\mathrm{E}_{R X}$ is calculated as follows,

$$
E_{\mathrm{TX}}(k, d)=E_{\text {elec }} * k+E_{\mathrm{amp}} * k^{*} d^{2}
$$

where $\mathrm{E}_{T X}(\mathrm{k}, \mathrm{d})$ is the overall energy needed to transmit a single ' $\mathrm{k}$ '-bit packet to a receiver over a distance ' $\mathrm{d}$ '. Also, $\mathrm{E}_{\text {elec }}$ is the transmit electronics, and $\mathrm{E}_{a m p}$ is the transmit amplifier.

$$
E_{R X}(k)=E_{\text {elec }} * k
$$

where $\mathrm{E}_{R X}(\mathrm{k})$ is the overall energy needed to receive a single ' $\mathrm{k}$ '-bit packet from a transmitter. ETALGOR follows the above energy model for each sensor node.

\section{Results and Discussion}

This section compares the proposed ETALGOR with LOADng applying ETX, N-ETX, RE, HC, PDR ratio, and energy consumed for packet retransmission. The ETALGOR intends to enhance the LOADng by including a metric N-ETX to decrease the extent of packet retransmission. This research uses composite measures to uncover the efficient path in the network: N-ETX and RE. Additional fields N-ETX and battery level parameters must report to each path. Figure 2 depicts a rudimentary IoT system.

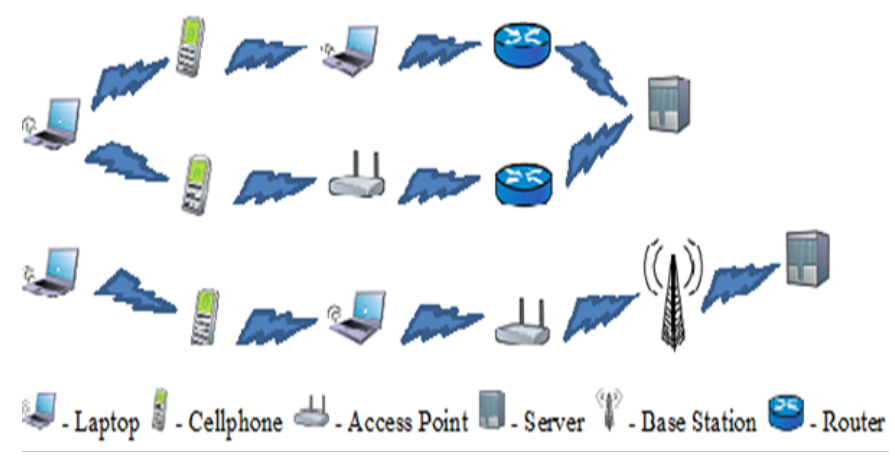

Fig 2. A Simple IoT Network

The N-ETX metric's accuracy determines path selection efficiency. N-ETX and RE measure the route quality. Consider the scenario in Figure 3, where the values on the edges represent a node's N-ETX and RE pair.

Five paths are available for information transfer from origin to the target node in the above scenario. They are $\mathrm{S} 1 \rightarrow \mathrm{S} 2 \rightarrow \mathrm{S} 3 \rightarrow \mathrm{S} 10, \mathrm{~S} 1 \rightarrow \mathrm{S} 4 \rightarrow \mathrm{S} 5 \rightarrow \mathrm{S} 10, \mathrm{~S} 1 \rightarrow \mathrm{S} 4 \rightarrow \mathrm{S} 5 \rightarrow \mathrm{S} 6 \rightarrow \mathrm{S} 10, \mathrm{~S} 1 \rightarrow \mathrm{S} 7 \rightarrow \mathrm{S} 8 \rightarrow \mathrm{S} 6 \rightarrow \mathrm{S} 10$ and $\mathrm{S} 1 \rightarrow \mathrm{S} 7 \rightarrow \mathrm{S} 8 \rightarrow \mathrm{S} 9 \rightarrow \mathrm{S} 10$. Path 1 has 3 hops, Path 2 has 3 hops, Path 3 has 4 hops, Path 4 has 4 hops, and Path 5 has 4 hops. The LOADng chooses the path $\mathrm{S} 1 \rightarrow \mathrm{S} 2 \rightarrow \mathrm{S} 3 \rightarrow \mathrm{S} 10$ because this has the fewest hops, i.e., hop count $=3$, by default as HC is the default metric for LOADng. But it ignored the upcoming dead nodes, and this path is not deemed energy efficient. This path is not deemed the optimum path by the ETALGOR because its RE is 250 Joules only. 


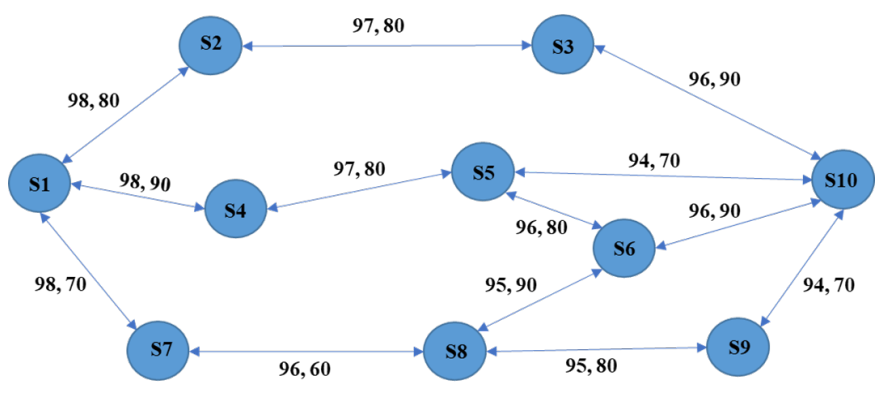

Fig 3. Routing Scenario of ETALGOR

The PDR ratio is determined to improve with implementing the N-ETX metric for the LOADng. The traditional ETX and the refined N-ETX by the ETALGOR are compared for various paths. Path $\mathrm{S} 1 \rightarrow \mathrm{S} 2 \rightarrow \mathrm{S} 3 \rightarrow \mathrm{S} 10$ yields ETX of $1.0309 \%$ whereas N-ETX achieves $1.0087 \%$, the path $\mathrm{S} 1 \rightarrow \mathrm{S} 4 \rightarrow \mathrm{S} 5 \rightarrow \mathrm{S} 10$ yields ETX of $1.0383 \%$ whereas N-ETX attains $1.0183 \%$. Path $\mathrm{S} 1 \rightarrow \mathrm{S} 4 \rightarrow \mathrm{S} 5 \rightarrow \mathrm{S} 6 \rightarrow \mathrm{S} 10$ produces $1.0336 \%$ while N-ETX produces $1.0148 \%$, the path $\mathrm{S} 1 \rightarrow \mathrm{S} 7 \rightarrow \mathrm{S} 8 \rightarrow \mathrm{S} 6 \rightarrow \mathrm{S} 10$ has ETX of $1.0418 \%$ while N-ETX yields $1.0289 \%$. The path $\mathrm{S} 1 \rightarrow \mathrm{S} 7 \rightarrow \mathrm{S} 8 \rightarrow \mathrm{S} 9 \rightarrow \mathrm{S} 10$ yields an ETX of $1.0446 \%$, whereas N-ETX produces $1.0358 \%$. The path with minimum N-ETX is the optimum path. It is seen from Table 2 the N-ETX is minimum for the path $\mathrm{S} 1 \rightarrow \mathrm{S} 4 \rightarrow \mathrm{S} 5 \rightarrow \mathrm{S} 6 \rightarrow \mathrm{S} 10$ which is deemed as the optimum energy-efficient path.

Table 2. Traditional ETX and N-ETX

\begin{tabular}{lll}
\hline Path $(\mathrm{s})$ & Traditional ETX (\%) & Refined N-ETX (\%) \\
\hline $\mathrm{S} 1 \rightarrow \mathrm{S} 2 \rightarrow \mathrm{S} 3 \rightarrow \mathrm{S} 10$ & 1.0309 & 1.0087 \\
$\mathrm{~S} 1 \rightarrow \mathrm{S} 4 \rightarrow \mathrm{S} 5 \rightarrow \mathrm{S} 10$ & 1.0383 & 1.0183 \\
$\mathrm{~S} 1 \rightarrow \mathrm{S} 4 \rightarrow \mathrm{S} 5 \rightarrow \mathrm{S} 6 \rightarrow \mathrm{S} 10$ & 1.0336 & 1.0148 \\
$\mathrm{~S} 1 \rightarrow \mathrm{S} 7 \rightarrow \mathrm{S} 8 \rightarrow \mathrm{S} 6 \rightarrow \mathrm{S} 10$ & 1.0418 & 1.0289 \\
$\mathrm{~S} 1 \rightarrow \mathrm{S} 7 \rightarrow \mathrm{S} 8 \rightarrow \mathrm{S} 9 \rightarrow \mathrm{S} 10$ & 1.0446 & 1.0358 \\
\hline
\end{tabular}

The table indicates that ETALGOR's N-ETX metric betters the traditional ETX measures for each path. This indicates that the ETALGOR delivers more packets than the LOADng.

Employing the ETALGOR, the path $\mathrm{S} 1 \rightarrow \mathrm{S} 4 \rightarrow \mathrm{S} 5 \rightarrow \mathrm{S} 6 \rightarrow \mathrm{S} 10$ is preferred as the energy-efficient path because it has the lowest N-ETX and the highest residual energy. To calculate N-ETX, sum up packet transmission and packet retransmission. The numerator signifies the quantity of packets accepted by a node; the denominator signifies the quantity of packets delivered from one node. Assume 100 packets from S1 to S10 are sent. Table 3 illustrates the packet transmission probability, variance, and ETX for LOADng. Here total node packets transmitted is $\mathrm{D}_{f}$ and received is $\mathrm{D}_{r}$.

The LOADng's variance $\left(\sigma^{2}\right)$ for $\mathrm{S} 1 \rightarrow \mathrm{S} 2 \rightarrow \mathrm{S} 3 \rightarrow \mathrm{S} 10$ path is 0.29 , and the ETX is 1.0309 , which is higher and results in needless packet retransmission as provided in Table 3.

Table 3. Variance and the ETX of the LOADng

\begin{tabular}{llllllll}
\hline Link(s) & Probability (\%) & Observed (O) & Expected $(\mathrm{E})$ & $(\mathrm{O}-\mathrm{E})$ & $(\mathrm{O}-\mathrm{E})^{2}$ & Variance $\sigma^{2}=(\mathrm{O}-\mathrm{E})^{2} / \mathrm{E}$ & $\mathrm{ETX}=\frac{1}{D_{f} * D_{r}}$ \\
\hline $\mathrm{S} 1 \rightarrow \mathrm{S} 2$ & 98 & 98 & 100 & -2 & 04 & $04 / 100=0.04$ & 1.0204 \\
$\mathrm{~S} 2 \rightarrow \mathrm{S} 3$ & 97 & 97 & 100 & -3 & 09 & $09 / 100=0.09$ & 1.0309 \\
$\mathrm{~S} 3 \rightarrow \mathrm{S} 10$ & 96 & 100 & -4 & 16 & $16 / 100=0.16$ & 1.0416 \\
& & & & & $\Sigma \sigma^{2}=0.29$ & Avg. ETX $=1.0309$ \\
\hline
\end{tabular}

The ETALGOR's variance $\left(\sigma^{2}\right)$ for $\mathrm{S} 1 \rightarrow \mathrm{S} 4 \rightarrow \mathrm{S} 5 \rightarrow \mathrm{S} 6 \rightarrow \mathrm{S} 10$ path is 0.13 , and the N-ETX is 1.0148 , which is optimum for the selected path than the LOADng's default path as provided in Table 4.

The ETALGOR's N-ETX measure enhances the PDR ratio from $98 \%$ to $99 \%$, from $97 \%$ to $98 \%$, and from $96 \%$ to $98 \%$ respectively for the links in the optimum path, compared with the LOADng's ETX PDR ratio of 97 and 96 percent. Table 5 indicates the ETALGOR's Degree of Variance using the T-test. The table confirms that the ETALGOR selects the most energyefficient path with N-ETX and RE. Its Degree of Variance is 0.13, and its RE is 340 Joules which is superior to other paths, while 
Table 4. Variance and N-ETX of the ETALGOR

\begin{tabular}{|c|c|c|c|c|c|c|c|}
\hline $\operatorname{Link}(s)$ & $\begin{array}{l}\text { Probability } \\
\text { (\%) }\end{array}$ & $\begin{array}{l}\text { Observed } \\
(\mathrm{O})\end{array}$ & Expected (E) & (O-E) & $(\mathrm{O}-\mathrm{E})^{2}$ & $\begin{array}{l}\text { Variance } \sigma^{2} \\
=(\mathrm{O}-\mathrm{E})^{2} / \mathrm{E}\end{array}$ & $\begin{array}{l}\text { N-ETX } \\
\frac{1}{\left(D_{f} * c\right)+\left((1-c) * D_{r}\right)}\end{array}$ \\
\hline $\mathrm{S} 1 \rightarrow \mathrm{S} 4$ & 99 & 99 & 100 & -1 & 01 & $\begin{array}{l}01 / 100= \\
0.01\end{array}$ & 1.0090 \\
\hline $\mathrm{S} 4 \rightarrow \mathrm{S} 5$ & 98 & 98 & 100 & -2 & 04 & $04 / 100=0.04$ & 1.0136 \\
\hline $\mathrm{S} 5 \rightarrow \mathrm{S} 6$ & 98 & 98 & 100 & -2 & 04 & $\begin{array}{l}04 / 100= \\
0.04\end{array}$ & 1.0183 \\
\hline \multirow[t]{2}{*}{$\mathrm{S} 6 \rightarrow \mathrm{S} 10$} & 98 & 98 & 100 & -2 & 04 & $\begin{array}{l}04 / 100= \\
0.04\end{array}$ & 1.0183 \\
\hline & & & & & & $\Sigma \sigma^{2}=0.13$ & $\begin{array}{l}\text { Avg. N-ETX }= \\
1.0148\end{array}$ \\
\hline
\end{tabular}

the Degree of Variance for LOADng is 0.45 . The path $\mathrm{S} 1 \rightarrow \mathrm{S} 4 \rightarrow \mathrm{S} 5 \rightarrow \mathrm{S} 6 \rightarrow \mathrm{S} 10$ is deemed the most optimum energy-efficient path that reduces unnecessary packet retransmission by the ETALGOR.

Table 5. Degree of Variance and RE

\begin{tabular}{|c|c|c|c|}
\hline Path(s) & Algorithms & $\begin{array}{l}\text { Degree of Variance } \Sigma \sigma^{2} \\
=(\mathrm{O}-\mathrm{E})^{2} / \mathrm{E}\end{array}$ & $\begin{array}{l}\text { Residual Energy(in } \\
\text { Joules) }\end{array}$ \\
\hline \multirow{2}{*}{$\mathrm{S} 1 \rightarrow \rightarrow \mathrm{S} 2 \rightarrow \mathrm{S} 3 \rightarrow \mathrm{S} 10$} & ETALGOR & 0.06 & \multirow{2}{*}{250} \\
\hline & LOADng & 0.29 & \\
\hline \multirow{2}{*}{$\mathrm{S} 1 \rightarrow \mathrm{S} 4 \rightarrow \mathrm{S} 5 \rightarrow \mathrm{S} 10$} & ETALGOR & 0.14 & \multirow{2}{*}{240} \\
\hline & LOADng & 0.49 & \\
\hline \multirow{2}{*}{$\mathrm{S} 1 \rightarrow \mathrm{S} 4 \rightarrow \mathrm{S} 5 \rightarrow \mathrm{S} 6 \rightarrow \mathrm{S} 10$} & ETALGOR & 0.13 & \multirow{2}{*}{340} \\
\hline & LOADng & 0.45 & \\
\hline \multirow{2}{*}{$\mathrm{S} 1 \rightarrow \mathrm{S} 7 \rightarrow \mathrm{S} 8 \rightarrow \mathrm{S} 6 \rightarrow \mathrm{S} 10$} & ETALGOR & 0.45 & \multirow{2}{*}{310} \\
\hline & LOADng & 0.70 & \\
\hline \multirow{2}{*}{$\mathrm{S} 1 \rightarrow \mathrm{S} 7 \rightarrow \mathrm{S} 8 \rightarrow \mathrm{S} 9 \rightarrow \mathrm{S} 10$} & ETALGOR & 0.70 & \multirow{2}{*}{280} \\
\hline & LOADng & 0.81 & \\
\hline
\end{tabular}

The path $\mathrm{S} 1 \rightarrow \mathrm{S} 2 \rightarrow \mathrm{S} 3 \rightarrow \mathrm{S} 10$ is more unpredictable, with $\sigma^{2}=0.29$ and the N-ETX is 1.0087 . However, this path has minimum N-ETX, this path is not selected as the optimum path by the ETALGOR because it fails with regard to RE, which is 250 Joules only, whereas the path $\mathrm{S} 1 \rightarrow \mathrm{S} 4 \rightarrow \mathrm{S} 5 \rightarrow \mathrm{S} 10$ has $\sigma^{2}=0.14$, and the N-ETX is 1.0183 , the path $\mathrm{S} 1 \rightarrow \mathrm{S} 7 \rightarrow \mathrm{S} 8 \rightarrow \mathrm{S} 6 \rightarrow \mathrm{S} 10$ has $\sigma^{2}=$ 0.45 , and the N-ETX is 1.0289 . The path $\mathrm{S} 1 \rightarrow \mathrm{S} 7 \rightarrow \mathrm{S} 8 \rightarrow \mathrm{S} 9 \rightarrow \mathrm{S} 10$ has $\sigma^{2}=0.70$, and the N-ETX $=1.0358$ is unpredictable.

The Degree of Independence " $t$ " of the ETALGOR is stated in Table 6, where the optimum path selected has a maximum N-ETX Variance $(\mathrm{t})$ of 0.0753 .

\begin{tabular}{ll}
\multicolumn{2}{l}{ Table 6. Degree of Independence of the ETALGOR } \\
\hline Path $(\mathbf{s})$ & $\mathrm{t}=\mathrm{N}-\mathrm{ETX}$ Variance \\
\hline $\mathrm{S} 1 \rightarrow \mathrm{S} 2 \rightarrow \mathrm{S3} \rightarrow \mathrm{S} 10$ & $\mathbf{0 . 0 6 6 7}$ \\
$\mathrm{S} 1 \rightarrow \mathrm{S} 4 \rightarrow \mathrm{S} 5 \rightarrow \mathrm{S} 10$ & $\mathbf{0 . 0 6 0 2}$ \\
$\mathrm{S} 1 \rightarrow \mathrm{S} 4 \rightarrow \mathrm{S} 5 \rightarrow \mathrm{S6} \rightarrow \mathrm{S} 10$ & $\mathbf{0 . 0 7 5 3}$ \\
$\mathrm{S} 1 \rightarrow \mathrm{S} 7 \rightarrow \mathrm{S} 8 \rightarrow \mathrm{S6} \rightarrow \mathrm{S} 10$ & $\mathbf{0 . 0 5 1 7}$ \\
$\mathrm{S} 1 \rightarrow \mathrm{S} 7 \rightarrow \mathrm{S} 8 \rightarrow \mathrm{S} 9 \rightarrow \mathrm{S} 10$ & $\mathbf{0 . 0 3 5 2}$ \\
\hline
\end{tabular}

The ETALGOR selects $\mathrm{S} 1 \rightarrow \mathrm{S} 4 \rightarrow \mathrm{S} 5 \rightarrow \mathrm{S} 6 \rightarrow \mathrm{S} 10$ path because this path has minimum N-ETX, maximum $\mathrm{RE}$, and maximum Degree of Independence " $t$ " which is evident from Table 4, Table 5, and Table 6, respectively.

hows the success probability of delivering packets, N-ETX, HC, and RE for the ETALGOR and theLOADng. The N-ETX ratio for the LOADng is $1.0309 \%, 1.0383 \%, 1.0336 \%, 1.0418 \%, 1.0446 \%$ respectively. The N-ETX ratio of $1.0087 \%, 1.0183 \%$, $1.0148,1.0289 \%, 1.0358 \%$ is accomplished by ETALGOR over LOADng, respectively. The PDR success probability for the LOADng is $97 \%, 96.33 \%, 96.75 \%, 96 \%, 95.75 \%$, respectively. The PDR success probability of $98.66 \%, 98 \%, 98.25 \%, 96.75 \%, 96 \%$ 
is accomplished by ETALGOR over LOADng, respectively. The optimum path $\mathrm{S} 1 \rightarrow \mathrm{S} 4 \rightarrow \mathrm{S} 5 \rightarrow \mathrm{S} 6 \rightarrow \mathrm{S} 10$ chosen by ETALGOR is having $\mathrm{N}-\mathrm{ETX}=1.0148, \mathrm{RE}=340$ Joules, and PDR success probability is $98.25 \%$ despite having 4 hops which are better than the LOADng with a PDR success probability of $96.75 \%$. The ETALGOR does not select path $\mathrm{S} 1 \rightarrow \mathrm{S} 2 \rightarrow \mathrm{S} 3 \rightarrow \mathrm{S} 10$ as the optimum path because it has $\mathrm{RE}=250$ Joules, which is smaller than the other paths, even though N-ETX and the HC is minimum because it causes a higher number of retransmission and re-route discovery when energy is completely depleted.

Table 7. Success probability, N-ETX, and RE of the ETALGOR and LOADng

\begin{tabular}{|c|c|c|c|c|c|}
\hline Path(s) & Algorithms & $\begin{array}{l}\text { N-ETX } \\
\frac{1}{\left(D_{f} * c\right)+\left((1-c) * D_{r}\right)} \\
(\%)\end{array}$ & $\begin{array}{l}\text { Success } \\
\text { Probability } \\
\text { (\%) }\end{array}$ & Hop Count & $\begin{array}{l}\text { Residual } \\
\text { Energy(in } \\
\text { Joules) }\end{array}$ \\
\hline \multirow{2}{*}{$\mathrm{S} 1 \rightarrow \mathrm{S} 2 \rightarrow \mathrm{S} 3 \rightarrow \mathrm{S} 10$} & ETALGOR & 1.0087 & 98.66 & \multirow{2}{*}{3} & \multirow{2}{*}{250} \\
\hline & LOADng & 1.0309 & 97 & & \\
\hline \multirow{2}{*}{$\mathrm{S} 1 \rightarrow \mathrm{S} 4 \rightarrow \mathrm{S} 5 \rightarrow \mathrm{S} 10$} & ETALGOR & 1.0183 & 98 & \multirow{2}{*}{3} & \multirow{2}{*}{240} \\
\hline & LOADng & 1.0383 & 96.33 & & \\
\hline \multirow{2}{*}{$\mathrm{S} 1 \rightarrow \mathrm{S} 4 \rightarrow \mathrm{S} 5 \rightarrow \mathrm{S6} \rightarrow \mathrm{S} 10$} & ETALGOR & 1.0148 & 98.25 & \multirow{2}{*}{4} & \multirow{2}{*}{340} \\
\hline & LOADng & 1.0336 & 96.75 & & \\
\hline \multirow{2}{*}{$\mathrm{S} 1 \rightarrow \mathrm{S} 7 \rightarrow \mathrm{S} 8 \rightarrow \mathrm{S6} \rightarrow \mathrm{S} 10$} & ETALGOR & 1.0289 & 96.75 & \multirow{2}{*}{4} & \multirow{2}{*}{310} \\
\hline & LOADng & 1.0418 & 96 & & \\
\hline \multirow{2}{*}{$\mathrm{S} 1 \rightarrow \mathbf{S} 7 \rightarrow \mathbf{S 8} \rightarrow \mathbf{S 9} \rightarrow \mathbf{S} 10$} & ETALGOR & 1.0358 & 96 & \multirow{2}{*}{4} & \multirow{2}{*}{280} \\
\hline & LOADng & 1.0446 & 95.75 & & \\
\hline
\end{tabular}

\section{POF of the ETALGOR}

The energy-efficient optimum path from the origin node to end node designated by the ETALGOR using the POF is given in equation (3),

$$
\operatorname{POF}\left(\sigma^{2}, t, \mathrm{RE}\right)=\operatorname{Min}\left\{\forall \sigma^{2}\right\} \cap \operatorname{Max}\{\forall t\} \cap \operatorname{Max}\{\forall F(R E)\}
$$

Suppose the node's RE is smaller than the $\mathrm{E}_{T H R}$ that node is rejected, where $\mathrm{E}_{T H R}$ is $5 \%$ of initial energy. This ensures forwarding packet(s) from origin to destination with minimal energy utilization for a node without the requirement for retransmission of packets. The function POF $\left(\sigma^{2}, t, R E\right)$ derived by the ETALGOR for an optimum path is indicated in equation (4) obtained from Table 5 and Table 6 , respectively,

$$
\operatorname{POF}\left(\sigma^{2}, \mathrm{t}, \mathrm{RE}\right)=\left\{\sigma^{2}=0.13\right\} \cap\{\mathrm{t}=0.0753\} \cap\{\mathrm{RE}=340 \text { Joules }\}
$$

In the ETALGOR, the cumulative total of all nodes RE exceeds the $\mathrm{E}_{T H R}$, hence the path $\mathrm{S} 1 \rightarrow \mathrm{S} 4 \rightarrow \mathrm{S} 5 \rightarrow \mathrm{S} 6 \rightarrow \mathrm{S} 10$ is deemed an energy-efficient path.

\section{Energy consumption of the ETALGOR}

The energy consumed per packet for the ETALGOR can be computed as follows: The time to transfer a single byte of the "DATA" packet is 0.611 milliseconds. The entire transmission time for an "ACK" packet is 0.304 milliseconds. The Chipcon CC-1312R ${ }^{(28)}$ is a low power wide supply voltage device that operates at voltages ranging from $1.8 \mathrm{~V}$ to $3.8 \mathrm{~V}$. It is used in real-world scenarios. Transmission Current (TX) current is $24.9 \mathrm{~mA}$, and Receiver Current (RX) current is $5.8 \mathrm{~mA}$ in the active transmission mode. Thus $\mathrm{TX}=24.9 \mathrm{~mA}=0.0249 \mathrm{~J}$ and the $\mathrm{RX}=5.8 \mathrm{~mA}=0.0058 \mathrm{~J}$.

To establish the best optimum path, the ETALGOR considers the three paths $\mathrm{S} 1 \rightarrow \mathrm{S} 4 \rightarrow \mathrm{S} 5 \rightarrow \mathrm{S} 6 \rightarrow \mathrm{S} 10, \mathrm{~S} 1 \rightarrow \mathrm{S} 7 \rightarrow \mathrm{S} 8 \rightarrow \mathrm{S} 6 \rightarrow \mathrm{S} 10$ and $\mathrm{S} 1 \rightarrow \mathrm{S} 7 \rightarrow \mathrm{S} 8 \rightarrow \mathrm{S} 9 \rightarrow \mathrm{S} 10$. As a magnitude of the factors, minimum N-ETX, maximum RE, and maximum Degree of Independence " $\mathrm{t}$ " the ETALGOR selects the path $\mathrm{S} 1 \rightarrow \mathrm{S} 4 \rightarrow \mathrm{S} 5 \rightarrow \mathrm{S} 6 \rightarrow \mathrm{S} 10$ as the optimum path. Table 8 signifies that the ETALGOR uses 11.2654 Joules while the LOADng uses 11.2959 Joules. The ETALGOR consumes 0.1964 Joules of energy to retransmit packets, while the LOADng consumes 0.3649 Joules. Here is a significant difference in energy use between the optimum path and the other options. Table 8 shows how much energy each route uses.

The ETALGOR excludes the paths $\mathrm{S} 1 \rightarrow \mathrm{S} 2 \rightarrow \mathrm{S} 3 \rightarrow \mathrm{S} 10$ and $\mathrm{S} 1 \rightarrow \mathrm{S} 4 \rightarrow \mathrm{S} 5 \rightarrow \mathrm{S} 10$ from the optimum path calculation since the RE of the two paths are 250 Joules and 240 Joules respectively, which is least significant compared to other paths. The ETALGOR uses 
Table 8. Energy consumed, RE, HC of the ETALGOR, and the LOADng

\begin{tabular}{|c|c|c|c|c|c|c|}
\hline Path(s) & Algorithms & $\begin{array}{l}\text { Energy consump- } \\
\text { tion for transmis- } \\
\text { sion of Packets (in } \\
\text { Joules) }\end{array}$ & $\begin{array}{l}\text { Energy con- } \\
\text { sumption for } \\
\text { retransmission } \\
\text { of Packets (in } \\
\text { Joules) }\end{array}$ & $\begin{array}{l}\text { Total energy spent } \\
\text { (in Joules) }\end{array}$ & $\begin{array}{l}\text { Residual } \\
\text { Energy (in } \\
\text { Joules) }\end{array}$ & $\begin{array}{l}\text { Hop } \\
\text { Count }\end{array}$ \\
\hline \multirow{2}{*}{$\mathrm{S} 1 \rightarrow \mathrm{S} 4 \rightarrow \mathrm{S} 5 \rightarrow \mathrm{S} 6 \rightarrow \mathrm{S} 10$} & ETALGOR & 11.069 & 0.1964 & 11.2654 & \multirow{2}{*}{340} & \multirow{2}{*}{4} \\
\hline & LOADng & 10.931 & 0.3649 & 11.2959 & & \\
\hline \multirow{2}{*}{$\mathrm{S} 1 \rightarrow \mathrm{S} 7 \rightarrow \mathrm{S} 8 \rightarrow \mathrm{S} 6 \rightarrow \mathrm{S} 10$} & ETALGOR & 10.9438 & 0.3369 & 11.2807 & \multirow{2}{*}{310} & \multirow{2}{*}{4} \\
\hline & LOADng & 10.8748 & 0.4212 & 11.296 & & \\
\hline \multirow{2}{*}{$\mathbf{S} 1 \rightarrow \mathbf{S} 7 \rightarrow \mathbf{S 8} \rightarrow \mathbf{S 9} \rightarrow \mathbf{S 1 0}$} & ETALGOR & 10.8618 & 0.4494 & 11.3112 & \multirow{2}{*}{280} & \multirow{2}{*}{4} \\
\hline & LOADng & 10.849 & 0.4773 & 11.3263 & & \\
\hline
\end{tabular}

the N-ETX metric and always considers eliminating dead nodes when selecting an optimum path. Those nodes are eliminated from inclusion into the routing table, contributing to further energy depletion later. Consequently, the node's power utilization is reduced while forwarding packets from one location to another. LOADng considers the shortest path with the smallest HC. Still, they don't consider factors like energy-depleted dead nodes, which means the routing table is re-configured repeatedly when the target node can't be reached, resulting in more energy consumption. ETALGOR and LOADng consume energy for packet retransmission. Still, ETALGOR consumes 0.1964 Joules, which is lower than LOADng's 0.3649 Joules since ETALGOR considers the N-ETX metric, which enhances the quality of the path, decreases the amount of packet retransmission, and increases network lifespan. The LOADng-IoT ${ }^{(12)}$ consumes 0.594 Joules for packet retransmission which is $15.2 \%$ additional energy than the ETALGOR. Figure 4 indicates the energy consumed by the retransmission of packets.

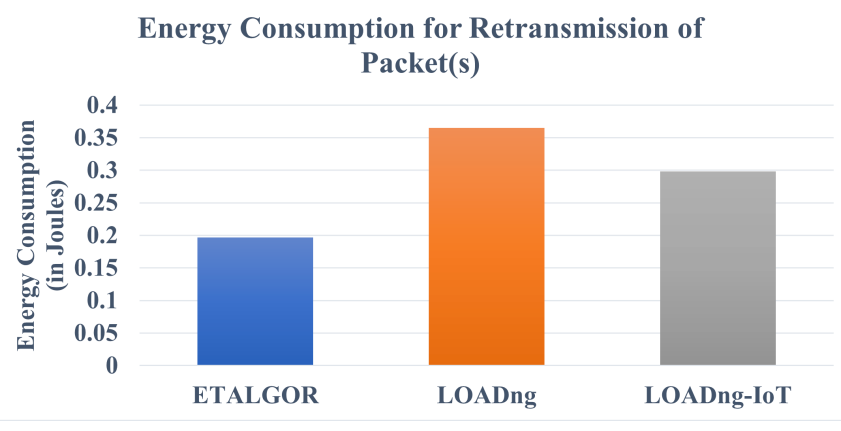

Fig 4. Energy consumed by ETALGOR for packet retransmission

\section{Simulation Results}

To test out the ETALGOR, the NS3 simulator is utilized to alter the LOADng to incorporate the N-ETX measure into the ETALGOR. The N-ETX metric and battery level are included in the ETALGOR control messages. In contrast to the LOADng, the ETALGOR employs the N-ETX and RE methods. The ETALGOR's performance was compared to the state-of-art protocols with respect to consumed energy, RE, PDR ratio, packet energy, and average energy consumption. Table 9 provides the simulation settings for the scenario indicated in Figure 3.

Figure 5 shows the simulation outcome of the ETALGOR with a snapshot for 25 nodes and 300 secs.

Experiments are carried out to determine how much energy ETALGOR uses compared to LOADng. As indicated in Figure 6 , it is evident that the ETALGOR consumes less energy than the LOADng. In the ETALGOR, fewer nodes are engaged in the packet forwarding process. The energy is saved much, as the path with ultimate POF $\left(\sigma^{2}, t, R E\right)$ always gets priority for packet forwarding. In the event of LOADng, more nodes forward packets, resulting in increased energy consumption. ETALGOR achieves an average energy proficiency improvement of 19.07\% over LOADng. An average energy consumption improvement of $11.04 \%$ is realized by the Genetic Algorithm based Ad hoc On-Demand Multipath Distance Vector routing protocol (GAAOMDV $)^{(5)}$. Thus, $15.80 \%$ better energy proficiency is achieved using ETALGOR over GA-AOMDV. The LOADng-IoT ${ }^{(12)}$ improves energy proficiency by $60 \%$ over the LOADng. Thus, $12.04 \%$ better energy proficiency is achieved using ETALGOR 
Table 9. Simulation Parameters

\begin{tabular}{ll}
\hline Parameters & Values \\
\hline No. of Nodes & 200 \\
Transmission Range & $500 \times 500 \mathrm{mts}$ \\
Simulation Time & 300 Secs \\
PHY/MAC & IEEE 802.15.4 \\
Channel & Wireless \\
Radio Propagation model & Matrix Propagation Loss Model \\
Radio Type & CC1312R \\
Application Type & CBR \\
Traffic Type & UDP \\
Packet Size & 512 Bytes \\
Transmission Power & 24.9 mA \\
Receiving Power & $5.8 \mathrm{~mA}$ \\
Initial Energy & 1000 Joules \\
Network Bandwidth & $2 \mathrm{Mbps}$ \\
Metrics & N-ETX, RE, HC \\
\hline
\end{tabular}

homepc@homepc: $\sim$ ns-allinone-3.28.1/bake/source/ns-3.28.1\$ ./waf --run scratch/loadng-etx-25node

Waf: Entering directory '/home/homepc/ns-allinone-3.28.1/bake/source/ns-3.28.1/build'

[2450/2902] Compiling scratch/loadng-etx-25node.cc

[2843/2902] Linking build/scratch/loadng-etx-25node

Waf: Leaving directory '/home/homepc/ns-allinone-3.28.1/bake/source/ns-3.28.1/build'

Build commands will be stored in build/compile_commands.json

'build' finished successfully $(10.808 \mathrm{~s})$

Cell voltage: 3V Cell Capacity: 1000J

End of simulation (300s) Total energy consumed by radio $=146.1468 \mathrm{~J}$

End of simulation (300s) Total energy consumed by radio $=135.1872 \mathrm{~J}$

End of simulation (300s) Total energy consumed by radio $=155.8416 \mathrm{~J}$

End of simulation $(300 \mathrm{~s})$ Total energy consumed by radio $=187.0392 \mathrm{~J}$

End of simulation (300s) Total energy consumed by radio $=203.5692 \mathrm{~J}$

End of simulation (300s) Total energy consumed by radio $=158.7798 \mathrm{~J}$

End of simulation (300s) Total energy consumed by radio $=141.3558 \mathrm{~J}$

End of simulation (300s) Total energy consumed by radio $=152.3682 \mathrm{~J}$

End of simulation (300s) Total energy consumed by radio $=172.4934 \mathrm{~J}$

End of simulation (300s) Total energy consumed by radio $=197.6628 \mathrm{~J}$

End of simulation (300s) Total energy consumed by radio $=229.488 \mathrm{~J}$

End

End of simulation (300s) Total energy consumed by radio $=206.4726 \mathrm{~J}$

End of

End

End of sim laion (300) Ton torgy consed by radio $=200.5674 \mathrm{~J}$

End of simulation (300) Tal nergy consumed by radio $=24.8708 \mathrm{~J}$

End of simulation (3005) Total encrgy consumed by radio $=214.6026 \mathrm{~J}$ End of simulation (300s) Total energy consumed by radio $=214.1086 \mathrm{~J}$ End of simulation (300s) Total energy consumed by radio $=213.2302 \mathrm{~J}$ End of simulation (300s) Total energy consumed by radio $=213.145 \mathrm{~J}$ End of simulation (300s) Total energy consumed by radio $=215.1036 \mathrm{~J}$ End of simulation (300s) Total energy consumed by radio $=214.8804 \mathrm{~J}$ End of simulation $(300 \mathrm{~s})$ Total energy consumed by radio $=214.8858 \mathrm{~J}$ End of simulation (300s) Total energy consumed by radio $=214.8678 \mathrm{~J}$ End of simulation (300s) Total energy consumed by radio $=245.0748 \mathrm{~J}$

Fig 5. NS3 Simulation outcome of the ETALGOR

compared to LOADng-IoT. The overall result reveals that ETALGOR is energy efficient irrespective of the volume of nodes.

Experiments are conducted to evaluate the network's RE of the ETALGOR over LOADng. Figure 7 indicates the network's total residual energy. ETALGOR achieves an average residual energy improvement of $10.51 \%$ over LOADng. An average residual energy improvement of $31.40 \%$ is attained by Intelligent Data Analytics-Optimized Energy Planning (IDA-OEP) protocol ${ }^{(1)}$. Thus, $15.43 \%$ better RE is accomplished using ETALGOR over IDA-OEP. The energy-efficient Optimal Multi-path Routing protocol (EOMR) ${ }^{(18)}$ improves residual energy by $15 \%$. Thus, $15.16 \%$ better RE is accomplished using ETALGOR compared to EOMR. The overall result achieved shows that ETALGOR has more RE than LOADng, IDA-OEP, and EOMR. It is noticeable that the RE decreases when the volume of nodes increases.

Experiments are conducted to evaluate the PDR ratio performance of ETALGOR over LOADng. The PDR ratio performance estimation is held by varying the volume of nodes in the network. A PDR ratio improvement of $98 \%, 97 \%, 96 \%, 94 \%, 94 \%$, $92 \%, 90 \%$ is accomplished by ETALGOR over LOADng considering 25, 50, 100, 200, 300, 400, 500 nodes respectively as 


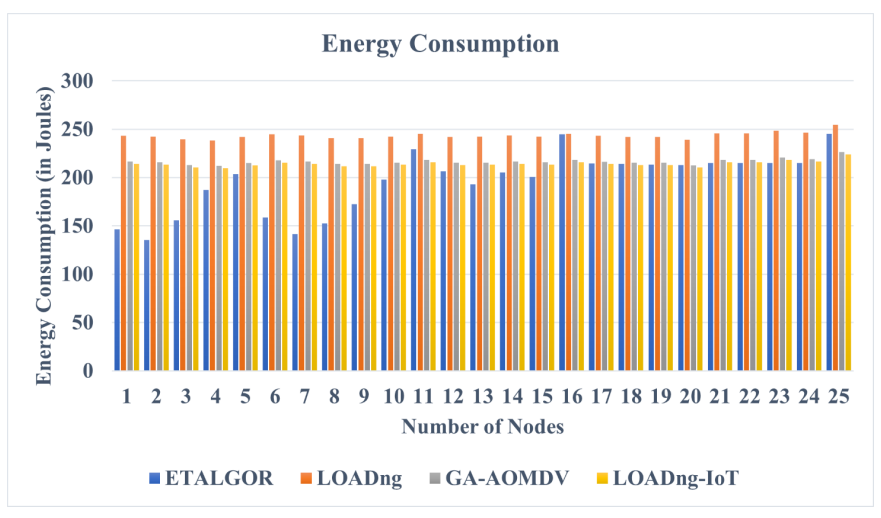

Fig 6. Energy consumption of ETALGOR

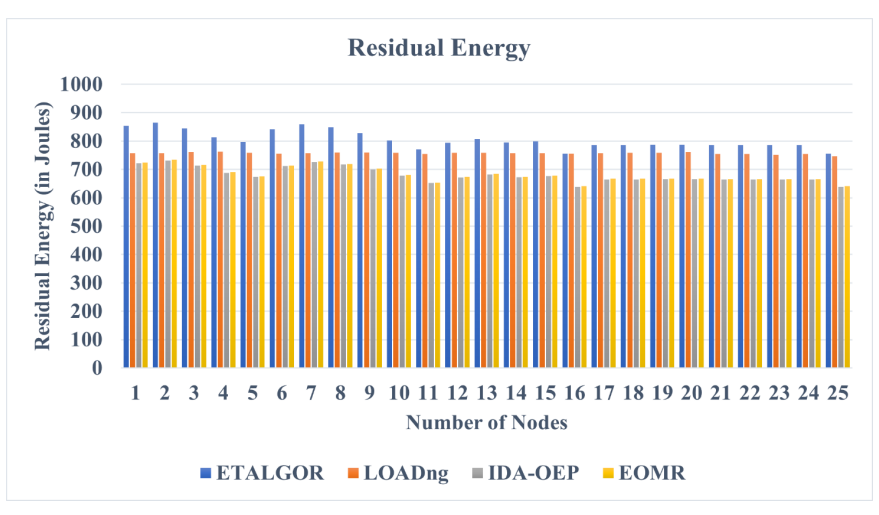

Fig 7. Network RE of ETALGOR

indicated in Figure 8. An overall PDR ratio for ETALGOR is improved by $10.58 \%$ over LOADng. A PDR ratio improvement of $12.86 \%$ is attained by the Reliable and Energy Efficient Route Selection algorithm (REERS) ${ }^{(7)}$. Thus, $10.2 \%$ better PDR ratio performance is accomplished by ETALGOR over REERS. The LOADng-IoT-Mob ${ }^{(11)}$ achieves a PDR ratio of $70 \%$ to $80 \%$ for all scenarios. Thus, ETALGOR achieves an overall improvement of 17.6\% PDR ratio over LOADng-IoT-Mob. The EOMR ${ }^{(18)}$ achieves a PDR ratio performance of $60 \%$ for parallel transmissions. The PDR ratio decreases with a rise in the volume of parallel transmissions. Thus, ETALGOR achieves $16.3 \%$ better PDR ratio performance over EOMR. Obviously, the ETALGOR outperforms the LOADng, REERS, and EOMR due to the inclusion of the N-ETX metric.

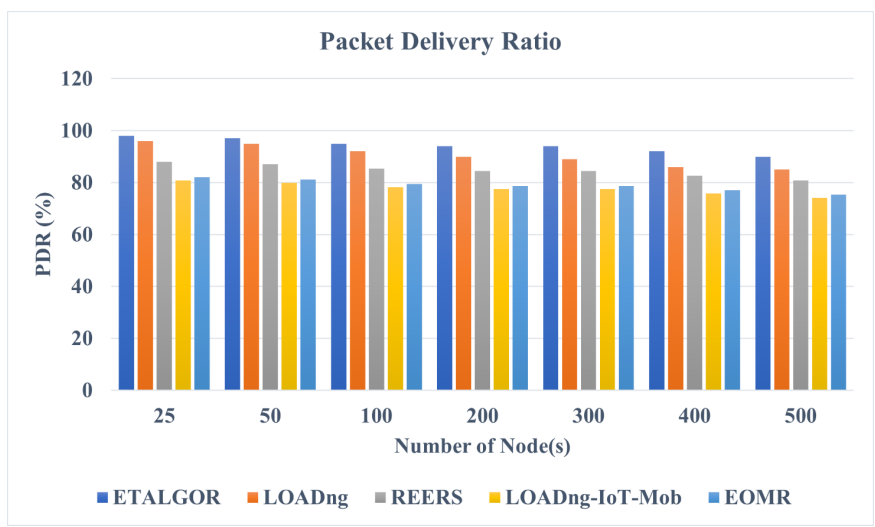

Fig 8. PDR ratio of ETALGOR 
Experiments are conducted to evaluate the network lifespan of ETALGOR over LOADng. Figure 9 indicates that the ETALGOR outperforms the LOADng in the perception of network lifespan. ETALGOR achieves an overall network lifetime increase of 30.54\% over LOADng. ETALGOR accomplishes an improvement of 7.69\% better lifetime over Energy Efficient Routing based on Reinforcement Learning (EER-RL) ${ }^{(6)}$. Network lifetime improvement of $71.64 \%$ is attained by REERS ${ }^{(7)}$. Thus, $5.80 \%$ better lifetime is accomplished using ETALGOR over REERS. ETALGOR achieves an overall lifetime improvement of $10.66 \%$ over EOMR ${ }^{(18)}$. ETALGOR achieves an overall lifetime improvement of $19.1 \%$ over ECRR ${ }^{(20)}$. Increased network life and battery lifespan are apparent because of the ETALGOR employed to discover the optimum path for information transmission. A node dies at any moment due to severe energy depletion if the current protocol is used as more nodes start transmitting data packets. In ETALGOR, only optimal nodes and paths are selected to send packets, increasing the network's lifetime and battery lifespan eliminating the frequent replacement of the battery.

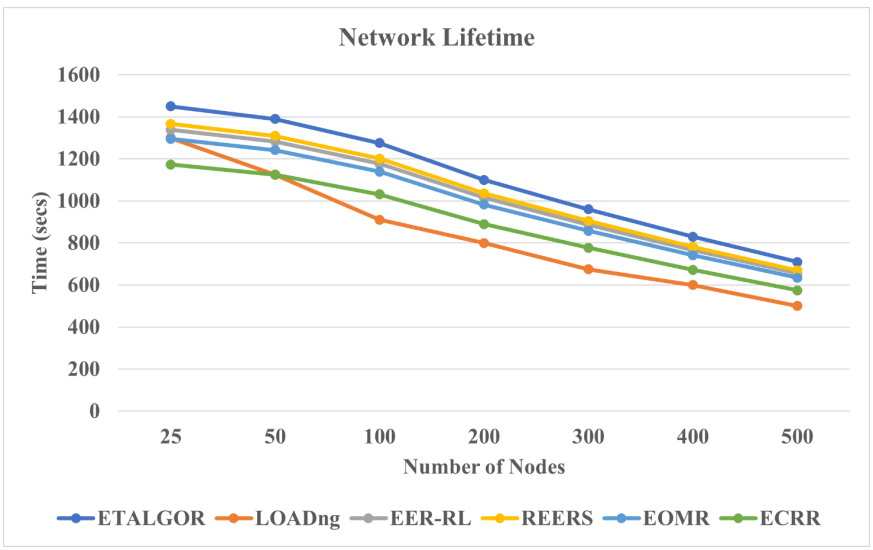

Fig 9. Network lifespan of ETALGOR

Additionally, the ETALGOR can be assessed with the volume of dropped packets, the packet failure ratio, end-to-end delay, latency, and throughput.

\section{Conclusion}

Energy utilization and minimizing node's energy consumption are significant problems in IoT. There are many packet collisions during neighbor node discovery. To limit the volume of packet retransmission, reduce energy usage, reduce packet failure, and increase network lifespan, ETALGOR is proposed. To choose the highly energy-efficient way to transfer packets, the proposed ETALGOR employs the metrics N-ETX, RE, and POF() function to find energy-efficient optimal paths. Experiments are conducted to evaluate the performance of the proposed ETALGOR over LOADng. The ETALGOR reduces energy utilization by $19.07 \%, 15.80 \%$, and $12.04 \%$, respectively, over LOADng, GA-AOMDV, and LOADng-IoT protocol. An average residual energy improvement of 10.51\%, 15.43\%, and 15.16\%, respectively, is accomplished by ETALGOR over LOADng, IDA-OEP, and EOMR protocol. The ETALGOR improves the PDR ratio to $10.58 \%, 10.2 \%, 17.6$, and 16.3\%, respectively, over LOADng, REERS, LOADng-IoT-Mob, and EOMR protocol. The ETALGOR enhances the network lifespan by 30.54\%, 7.69\%, 5.80\%, 10.66\%, and 19.1\%, respectively, over LOADng, EER-RL, REERS, EOMR, and ECRR protocol. A node consumes less energy and takes less effort to retransmit packets when the ETALGOR is used. It also attempts to discover the best energy-efficient path for transmission of packets by selecting paths with low N-ETX and high residual energy to prevent retransmission of packets. Performance evaluation demonstrates that energy consumed by the network and the node is reduced, PDR ratio increased, energy consumption per node decreased, and balanced residual energy between nodes signifies that the ETALGOR outperforms the LOADng and other state-of-art protocols.

Future work would consider the paths and nodes with unbalanced ETX links. These nodes will be overloaded more rapidly, requiring more time to process the packets, high memory, and bandwidth, causing more energy exhaustion and increased latency, and failing to capture the link reliability. Also, future work can be augmented by selecting multiple energy-efficient optimum paths to decrease unnecessary packet retransmission and reduce latency. 


\section{References}

1) Arul R, Alroobaea R, Mechti S, Rubaiee S, Andejany M, Tariq U, et al. Intelligent data analytics in energy optimization for the internet of underwater things. Soft Computing. 2021;25(18):12507-12519. Available from: https://dx.doi.org/10.1007/s00500-021-06002-x.

2) Biradar M, Mathapathi B. Secure, Reliable and Energy Efficient Routing in WSN: A Systematic Literature Survey. 2021 International Conference on Advances in Electrical, Computing, Communication and Sustainable Technologies (ICAECT). 2021. Available from: https://doi.org/10.1109/icaect49130. 2021.9392561.

3) Zanaj E, Caso G, Nardis LD, Mohammadpour A, Alay Ö, Benedetto MGD. Energy Efficiency in Short and Wide-Area IoT Technologies-A Survey. Technologies. 2021;9(1):22-22. Available from: https://dx.doi.org/10.3390/technologies 9010022 .

4) Khan MZ, Alhazmi OH, Javed MA, Ghandorh H, Aloufi KS. Reliable Internet of Things: Challenges and Future Trends. Electronics. 2021;10(19):23772377. Available from: https://dx.doi.org/10.3390/electronics10192377.

5) Patel J, El-Ocla H. Energy Efficient Routing Protocol in Sensor Networks Using Genetic Algorithm. Sensors. 2021;21(21):7060-7060. Available from: https://dx.doi.org/10.3390/s21217060.

6) Mutombo VK, Lee S, Lee J, Hong J. EER-RL: Energy-Efficient Routing Based on Reinforcement Learning. Mobile Information Systems. 2021;2021:1-12. Available from: https://doi.org/10.1155/2021/5589145.

7) Nayagi DS, G SG, Ravi V, R VK, Sennan S. REERS: Reliable and energy-efficient route selection algorithm for heterogeneous Internet of things applications. International Journal of Communication Systems. 2021;34(13). Available from: https://dx.doi.org/10.1002/dac.4900.

8) Zhang S, You X, Zhang P, Huang M, Li S. A UCB-based dynamic CoAP mode selection algorithm in distribution IoT. Alexandria Engineering Journal. 2022;61(1):719-727. Available from: https://dx.doi.org/10.1016/j.aej.2021.04.101.

9) Clausen T, Yi J, A C. The Lightweight On-demand Ad hoc Distance-vector Routing Protocol - Next Generation (LOADng). . Available from: https://datatracker.ietf.org/doc/html/draft-clausen-lln-loadng-15.

10) Zikria YB, Afzal MK, Ishmanov F, Kim SW, Yu H. A survey on routing protocols supported by the Contiki Internet of things operating system. Future Generation Computer Systems. 2018;82:200-219. Available from: https://dx.doi.org/10.1016/j.future.2017.12.045.

11) Sobral JVV, Rodrigues JJPC, Rabelo RAL, Saleem K, Kozlov SA. Improving the Performance of LOADng Routing Protocol in Mobile IoT Scenarios. IEEE Access. 2019;7:107032-107046. Available from: https://dx.doi.org/10.1109/access.2019.2932718.

12) Sobral JVV, Rodrigues JJPC, Rabêlo RAL, Saleem K, Furtado V. LOADng-IoT: An Enhanced Routing Protocol for Internet of Things Applications over Low Power Networks. Sensors. 2019;19(1):150-150. Available from: https://dx.doi.org/10.3390/s19010150.

13) Halepoto IA, Khan UA, Arain AA. Retransmission Policies for Efficient Communication in IoT Applications. 2018 IEEE 6th International Conference on Future Internet of Things and Cloud (FiCloud). 2018. Available from: https://doi.org/10.1109/ficloud.2018.00036.

14) Paul B. A Novel Mathematical Model to Evaluate the Impact of Packet Retransmissions in LoRaWAN. IEEE Sensors Letters. 2020;4(5):1-4. Available from: https://doi.org/10.1109/lsens.2020.2986794.

15) Zhang X, Qi H, Zhang X, Han L. Energy-Efficient Resource Allocation and Data Transmission of Cell-Free Internet of Things. IEEE Internet of Things Journal. 2021;8(20):15107-15116. Available from: https://dx.doi.org/10.1109/jiot.2020.3030675.

16) Ansere JA, Han G, Bonsu KA, Peng Y. Energy-Efficient Joint Power Allocation and User Selection Algorithm for Data Transmission in Internet-of-Things Networks. IEEE Internet of Things Journal. 2020;7(9):8736-8747. Available from: https://dx.doi.org/10.1109/jiot.2020.2995387.

17) Fathy Y, Barnaghi P. Quality-Based and Energy-Efficient Data Communication for the Internet of Things Networks. IEEE Internet of Things Journal. 2019;6(6):10318-10331. Available from: https://dx.doi.org/10.1109/jiot.2019.2938101.

18) Izaddoost A, Siewierski M. Energy Efficient Data Transmission in IoT Platforms. Procedia Computer Science. 2020;175:387-394. Available from: https://dx.doi.org/10.1016/j.procs.2020.07.055.

19) Khan RA, Xin Q, Roshan N. RK-Energy Efficient Routing Protocol for Wireless Body Area Sensor Networks. Wireless Personal Communications. 2021;116(1):709-721. Available from: https://dx.doi.org/10.1007/s11277-020-07734-z.

20) Praveen KV, Prathap PMJ. Energy Efficient Congestion Aware Resource Allocation and Routing Protocol for IoT Network using Hybrid Optimization Techniques. Wireless Personal Communications. 2021;117(2):1187-1207. Available from: https://dx.doi.org/10.1007/s11277-020-07917-8.

21) Ahad A, Tahir M, Sheikh MA, Ahmed KI, Mughees A, Numani A. Technologies Trend towards 5G Network for Smart Health-Care Using IoT: A Review. Sensors. 2020;20(14):4047-4047. Available from: https://dx.doi.org/10.3390/s20144047.

22) Anjos JCSD, Gross JLG, Matteussi KJ, González GV, Leithardt VRQ, Geyer CFR. An Algorithm to Minimize Energy Consumption and Elapsed Time for IoT Workloads in a Hybrid Architecture. Sensors. 2021;21(9):2914-2914. Available from: https://dx.doi.org/10.3390/s21092914.

23) Jaiswal K, Anand V. EOMR: An Energy-Efficient Optimal Multi-path Routing Protocol to Improve QoS in Wireless Sensor Network for IoT Applications. Wireless Personal Communications. 2020;111(4):2493-2515. Available from: https://dx.doi.org/10.1007/s11277-019-07000-x.

24) Nivedhitha V, Saminathan AG, Thirumurugan P. DMEERP: A dynamic multi-hop energy efficient routing protocol for WSN. Microprocessors and Microsystems. 2020;79:103291-103291. Available from: https://dx.doi.org/10.1016/j.micpro.2020.103291.

25) Maheshwari P, Sharma AK, Verma K. Energy efficient cluster based routing protocol for WSN using butterfly optimization algorithm and ant colony optimization. Ad Hoc Networks. 2021;110:102317-102317. Available from: https://dx.doi.org/10.1016/j.adhoc.2020.102317.

26) Sujanthi S, Kalyani SN. SecDL: QoS-Aware Secure Deep Learning Approach for Dynamic Cluster-Based Routing in WSN Assisted IoT. Wireless Personal Communications. 2020;114(3):2135-2169. Available from: https://dx.doi.org/10.1007/s11277-020-07469-x.

27) Shende DK, Sonavane SS. CrowWhale-ETR: CrowWhale optimization algorithm for energy and trust aware multicast routing in WSN for IoT applications. Wireless Networks. 2020;26(6):4011-4029. Available from: https://dx.doi.org/10.1007/s11276-020-02299-y.

28) SimpleLink ${ }^{\mathrm{TM}}$ 32-bit Arm Cortex-M4F Sub-1 GHz wireless MCU for IEEE 802.15.4, 6LoWPAN. . Available from: https://www.ti.com/product/CC1312R. 